\title{
The prenatal diagnosis and clinical outcomes of fetuses with 15q11.2 copy number variants: a case series of 36 patients
}

\author{
Jessica Kang ${ }^{1}$, Chien Nan Lee ${ }^{2}$, Yi-Ning Su${ }^{3}$, Ming-Wei Lin ${ }^{4}$, Yi-Yun Tai ${ }^{2}$, Wen wei Hsu ${ }^{2}$, \\ KUAN-YING HUANG ${ }^{4}$, Chi-Ling Chen ${ }^{2}$, Chien-Hui Hung ${ }^{2}$, and SHIN-YU LIN ${ }^{2}$ \\ ${ }^{1}$ National Taiwan University Hospital, National Taiwan University College of Medicine \\ ${ }^{2}$ National Taiwan University Hospital \\ ${ }^{3}$ Dianthus MFM Center \\ ${ }^{4}$ National Taiwan University Hospital Hsin-Chu Branch
}

April 28, 2021

\begin{abstract}
Objective: The prenatal genetic counseling of fetus diagnosed with the 15q11.2 copy number variant (CNV) involving the BP1-BP2 region has been difficult due to limited information and controversial opinion on prognosis. Design: Case series. Setting: This study uses data from National Taiwan University Hospital. Sample: Data of 36 pregnant women who underwent prenatal microarray analysis from 2012 to 2017 and were assessed at National Taiwan University Hospital. Methods: Data were collected by reviewing patients' medical record. Comparison of patient characteristics, prenatal ultrasound findings and postnatal outcomes between different cases involving the 15q11.2 BP1-BP2 region were presented. Main outcome measured: Postnatal prognosis. Results: Out of the 36 patients diagnosed with CNVs involving the BP1-BP2 region, 5 were diagnosed with microduplication and 31 with microdeletion. Abnormal ultrasound findings were recorded in 12 cases prenatally. De novo microduplications were observed in $25 \%$ of the cases and microdeletions were found in $14 \%$. Amongst the cases, 10 pregnant women received termination of pregnancy and 26 gave birth to healthy individuals (27 babies in total). Conclusion: The prognoses of 15q11.2 CNVs were controversial and recent studies have revealed its connection with developmental delay and autism. In our study, no obvious developmental delay or neurological disorders were detected postnatally in the 1 case of 15 q11.2 microduplication and 25 cases of microdeletion.
\end{abstract}

\section{Hosted file}

15q11.2-main.pdf available at https://authorea.com/users/346431/articles/519855-the-prenataldiagnosis-and-clinical-outcomes-of-fetuses-with-15q11-2-copy-number-variants-a-caseseries-of-36-patients 


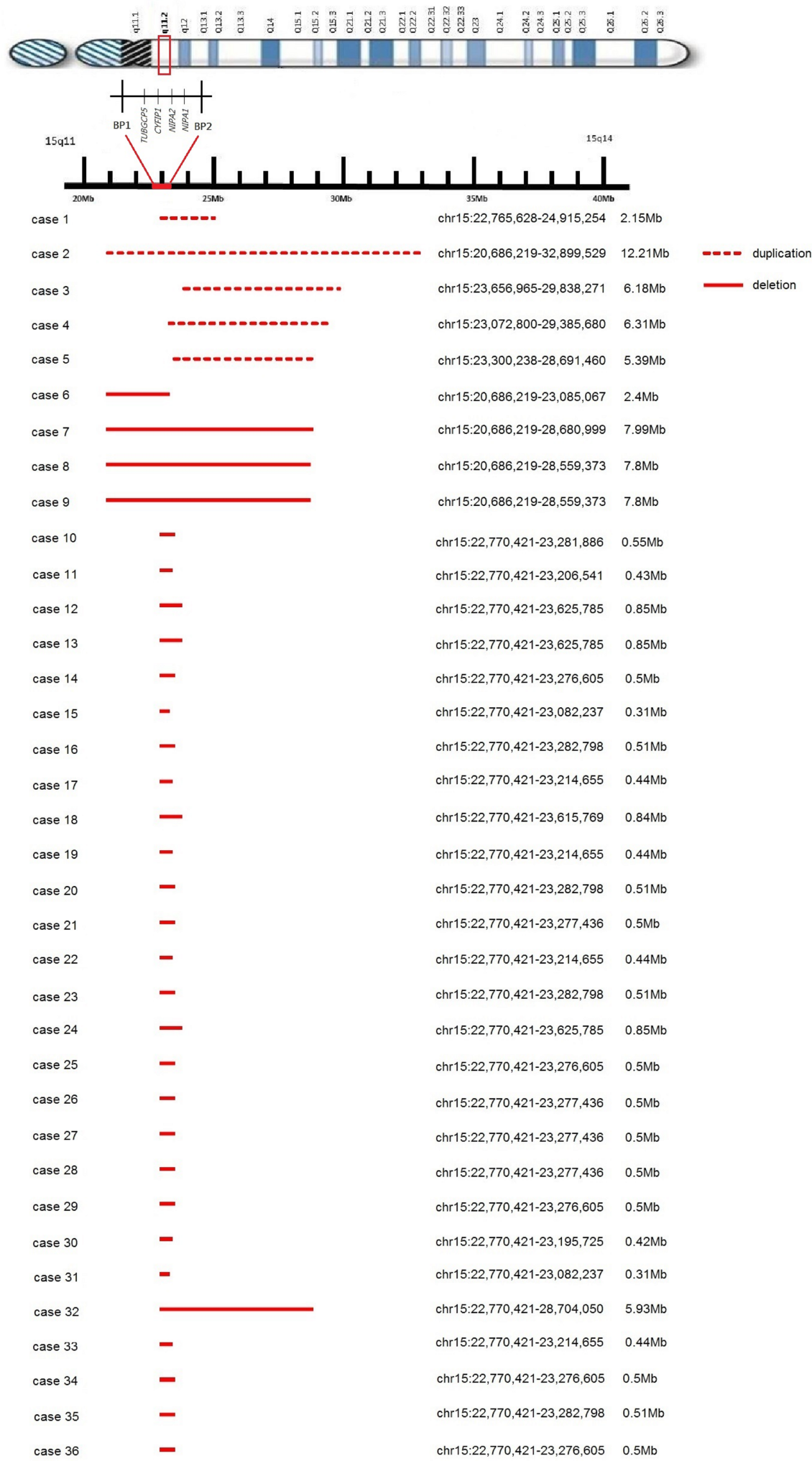

